\title{
"Acute Primary Total Hip Arthroplasty for Combined Posterior Acetabulum Fracture with Ipsilateral Associated Posteriorly Dislocated Femoral Head with Femoral Neck Fracture. Using of Femoral Head as an Autograft Would be an Advantage"
}

\author{
Babaji Thorat $1^{1}$, Avtar Singh ${ }^{1}$, Mohammad Arshad ${ }^{1}$, Sharad Salokhe ${ }^{1}$, Ravi Mavani $^{1}$
}

Learning Point of the Article:

Acetabular reconstruction using femoral head structural autograft and acute primary uncemented THA is a viable alternative to internal fixation in middle-aged patients with comminuted fracture of the acetabulum and femoral neck with hip dislocation.

\section{Abstract}

Introduction: Traumatic posterior hip dislocation with comminuted fracture of the ipsilateral acetabulum and femoral neck is a rare fracture pattern. These injuries are associated with high energy trauma and pose challenges during management. Controversy exists between hip preservation and replacement surgeries in middle-age patients. Open reduction and internal fixation (ORIF) have a high risk of non-union, avascular necrosis, and post-traumatic osteoarthritis of hip requiring total hip arthroplasty hip replacement (THA) as a secondary procedure later.

Case Report: A 56-year-old male presented with posterior hip dislocation and comminuted fracture of ipsilateral wall and column of the acetabulum, and femoral neck following a high energy trauma. He was managed by acetabular reconstruction using femoral head structural autograft combined with acute primary uncemented THA. At 2-year follow-up, the patient had good functional outcome with a satisfactory range of motion without any difficulty in weight-bearing and doing his daily activities.

Conclusion: Although uncommon, acetabular reconstruction using femoral head structural autograft and acute primary uncemented THA is a viable alternative treatment option compared to ORIF in middle-age patients with fracture of ipsilateral neck and acetabulum. This facilitates post-operative rehabilitation and avoids further operations for possible developing AVN or secondary arthritis.

Keywords: Hip dislocation, acetabulum fracture, femur neck fracture, acute total hip arthroplasty, acetabular reconstruction, femoral head structural autograft.

\section{Introduction}

Posterior hip dislocations account for $90 \%$ of all hip dislocations. Ipsilateral acetabular fractures are associated in up to $30 \%$ of posterior hip dislocations, typically caused by a high energy trauma and usually have concomitant injuries [1].

The Previous previous studies have reported significant morbidity and poor functional outcome with open reduction and internal fixation (ORIF) owing due to post-traumatic avascular necrosis (AVN) and secondary osteoarthritis of hip, necessitating total hip arthroplasty (THA) as a secondary procedure in near future [2]. Primary THA is recommended by some authors for such injuries in elderly patients [3]. However, primary THA with acetabular reconstruction in acute setting is a complex and demanding procedure with unpredictable outcomes due to distorted anatomy, fracture comminution posing problems during implantation $[3,4]$. Therefore, a dilemma still exists whether to preserve or sacrifice the femoral head as decision making becomes more difficult in patients in their 4 th or 5 th decades of life with limited literature available to

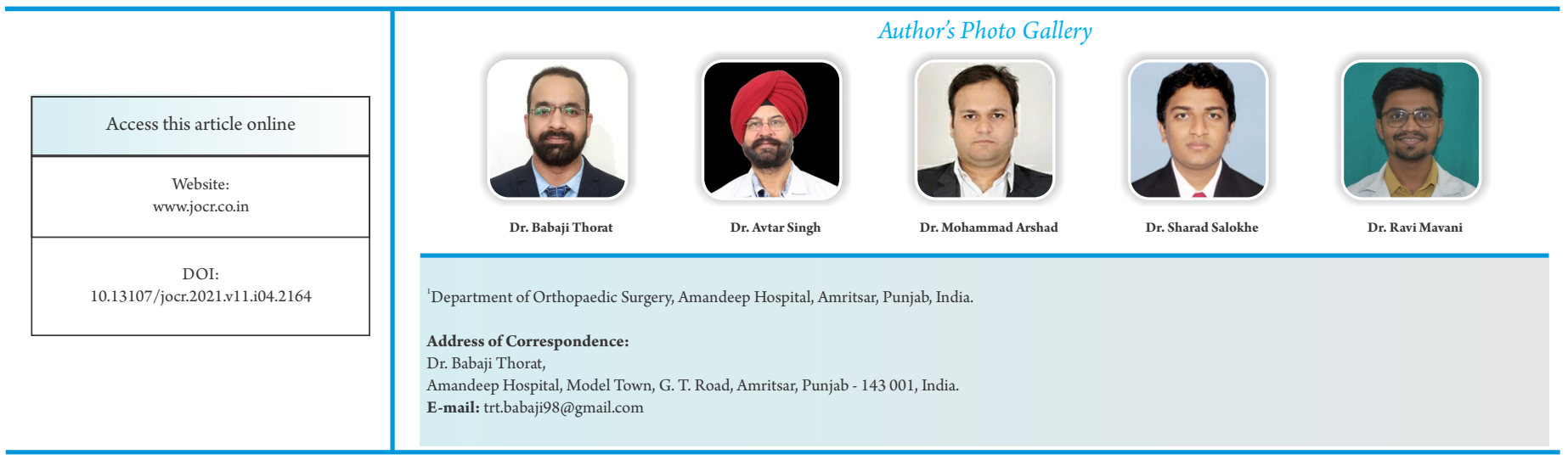

Journal of Orthopaedic Case Reports | pISSN 2250-0685 | eISSN 2321-3817 | Available on www.jocr.co.in | doi:10.13107/jocr.2021.v11.i04.2164 This is an Open Access article distributed under the terms of the Creative Commons Attribution Non-Commercial License (http://creativecommons.org/licenses/by-nc/3.0) which permits unrestricted non-commercial use, distribution, and reproduction in any medium, provided the original work is properly cited. 


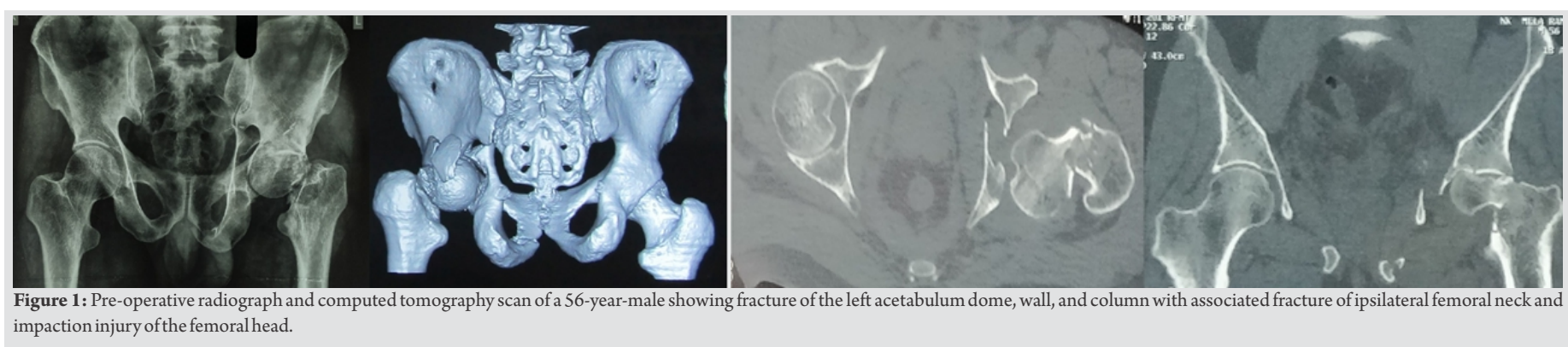

support the definitive treatment option [1].

Moreover, the combination of ipsilateral comminuted fracture of wall and column of acetabulum and femoral neck with posterior hip dislocation is an infrequently reported injury as the mechanism of both these injuries is not similar. To the best of our knowledge, early management of such injury combination by acetabular reconstruction using femoral head structural autograft and acute uncemented primary THA is rarely reported in literature till date. We, hereby, present a case with above combination and detailed discussion on surgical challenges.

\section{Case Report}

A 56-year-old male was brought to the emergency department complaining of severe pain and swelling in the left hip region with a history of a car accident with the patient sitting in the passenger seat, with his hip and knee flexed such as in a dashboard injury. On physical examination, he was conscious and oriented, but, unable to straight leg raise of the left lower limb which was slightly internally rotated, flexed, and shortened as compared to the opposite uninjured limb. Palpation revealed tenderness in pubic and left hip region. Radiographs and computerized tomography (Fig. 1) scan showed posterior dislocation of the left hip with comminuted fracture of ipsilateral acetabular dome, wall and column, and femoral neck. The patient was neurovascularly intact without any additional injuries. Pre-operative workup, counselling and written informed consent were done regarding the nature of the injury, treatment options, and complications.
He underwent surgery in lateral decubitus position using posterior approach. Intraoperatively, short external rotators of hip found are torn with ragged margins and an intact sciatic nerve. Fracture neck femur (Pauwel type 2) was having posterosuperior comminution and the fracture line was found to be extending basicervical neck to the base of the head of the femur with impaction articular cartilage injury of the femoral head. Acetabular posterior wall and column fracture was were comminuted with loose wall fragments. The posterior column was found displaced posteromedially and reduced with the help of pointed reduction pelvic clamps. Stable definitive fixation of posterior column of the acetabulum was performed using 8hole $3.5 \mathrm{~mm}$ titanium reconstruction plate and interfragmentary screws. Then, the fracture geometry of acetabulum was redefined. The femoral head was used to reconstruct the deficient posterior wall and dome of the acetabulum, which was provisionally fixed with $\mathrm{k}$-wire and then secured by two $6.5 \mathrm{~mm}$ partial thread cancellous screw and one $3.5 \mathrm{~mm}$ cortical screw (Fig. 2). Using the standard technique, an acute primary uncemented THA was performed using $60 \mathrm{~mm}$ porous acetabular metal shell, liner ( $60 \mathrm{~mm}$ O.D), $36 \mathrm{~mm}$ femoral head, $60 \mathrm{~mm}$ (O.D), and size 7 femoral short stem. The acetabular component was fixed with screws for additional support. The hip was stable in all direction after reduction. The operating time was $80 \mathrm{~min}$ and the intraoperative blood loss was $550 \mathrm{~mL}$.

Post-operative period was uneventful with satisfactory component alignment on radiographs (Fig. 3). He was kept non-weight bearing for 1 month followed by gradually increase in weight-bearing with support. The patient was able to bear full weight without support and radiographs showed a healed

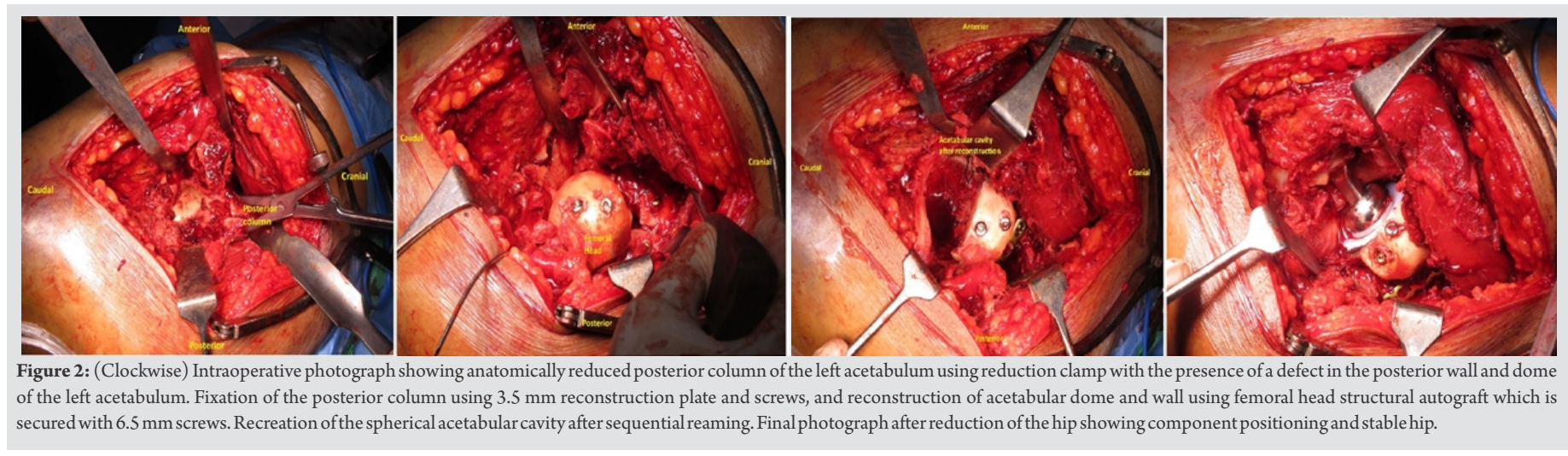




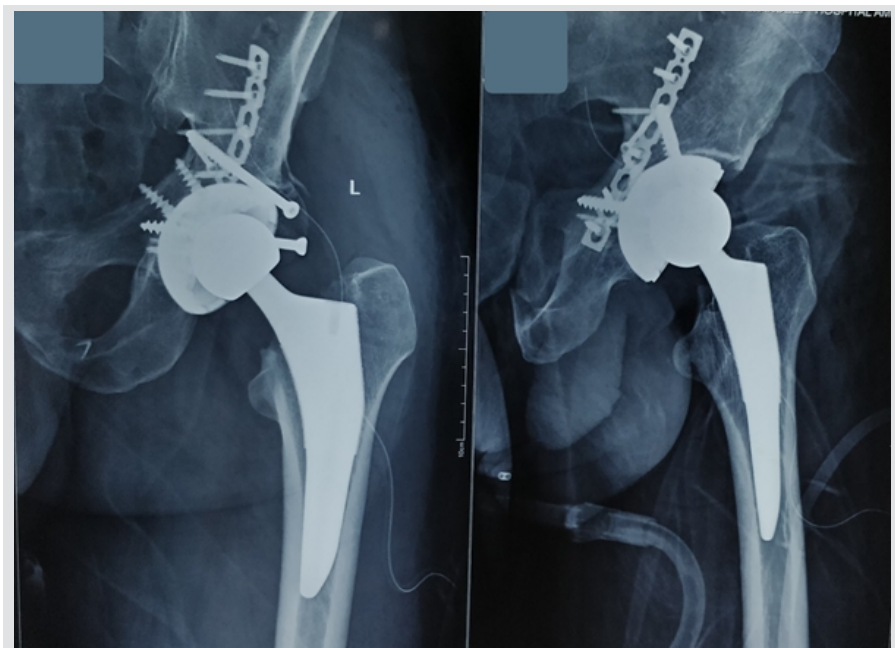

Figure 3: Immediate post-operative radiograph after acute primary uncemented total hip arthroplasty of the left hip with acetabular reconstruction using femoral head structural autograft showing anatomical reduction of the acetabular fracture along with satisfactory alignment and positioning of the femoral and acetabular components.

fracture with stable implant at 6-month follow-up (Fig. 4). At 2year follow-up, he had a good hip function (Fig. 5) a Harris Hip Score of 90.8 with a stable well-fixed implant [2] (Fig. 6).

\section{Discussion}

An ipsilateral comminuted fracture of the femoral neck, acetabular posterior wall and column with hip dislocation is are essentially a rare injury, seen usually in young patients with $75 \%$ of them aged $<50$ years [1]. Deciding between femoral head preservation orand replacement surgery remains controversial due to rarity of injury pattern, the paucity of a definite classification system and lack of consensus regarding management [1]. The final clinical outcome depends on the interval between the time of injury and treatment, type of surgical intervention, and implant selection.

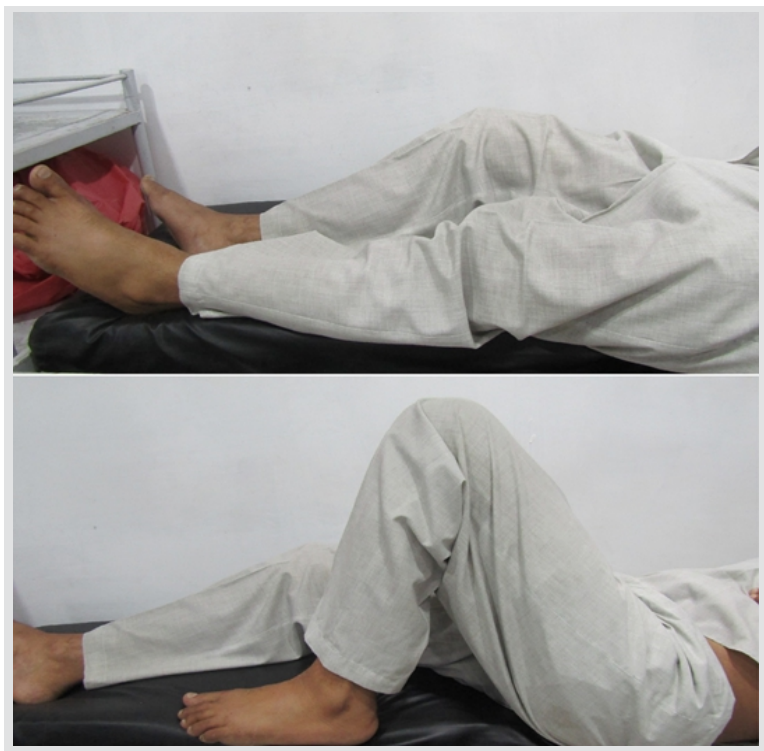

Figure 5: Clinical photographs of the left hip showing good hip function and joint mobility at final follow-up. osteolysis.

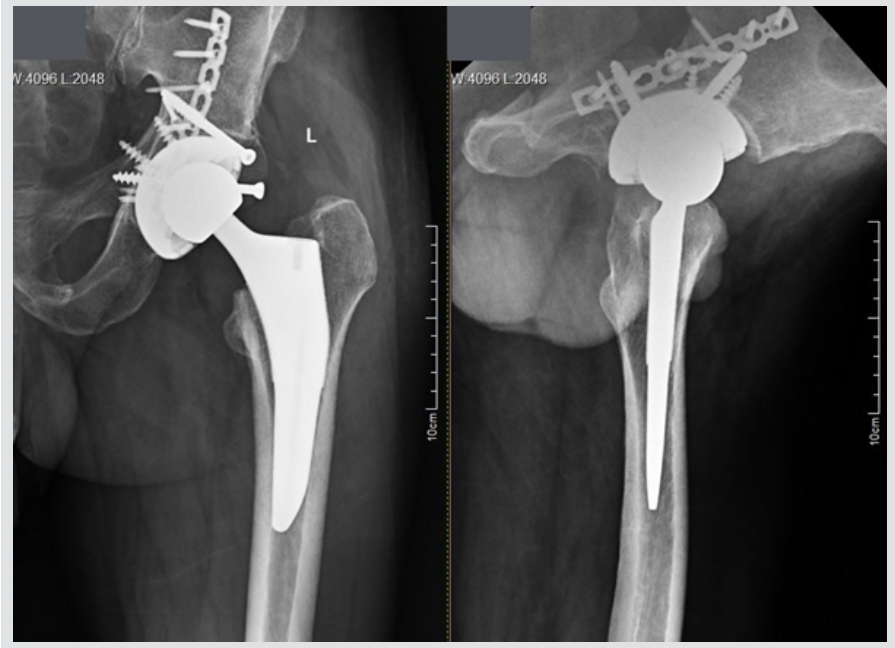

Figure 4: Anteroposterior and lateral radiograph of left hip showing healed fracture with stable total hip arthroplasty in situ.

Some studies recommend preservation of femoral head, especially in young patients, as it restores the natural bone stock of the patient and avoids complication related to acute arthroplasty such as dislocation, prosthesis loosening $[1,5]$. However, in certain patients with complex fracture pattern, survival of the femoral head is doubtful and the fracture is not amenable for fixation, and an unfavourable outcome such as AVNavascular necrosis, post-traumatic osteoarthritis, or nonunion is anticipated in up- to $13-67 \%$ patients requiring conversion into an arthroplasty in near future regardless of treatment $[3,5,6,7,8]$.

Therefore, it is worthwhile to perform acute THA as a definitive treatment in these cases as it leads to early restoration of function, improved survival with a reduced period of immobilization and prevents secondary complications $[7,8]$. The Pprevious discouraging results after acute primary

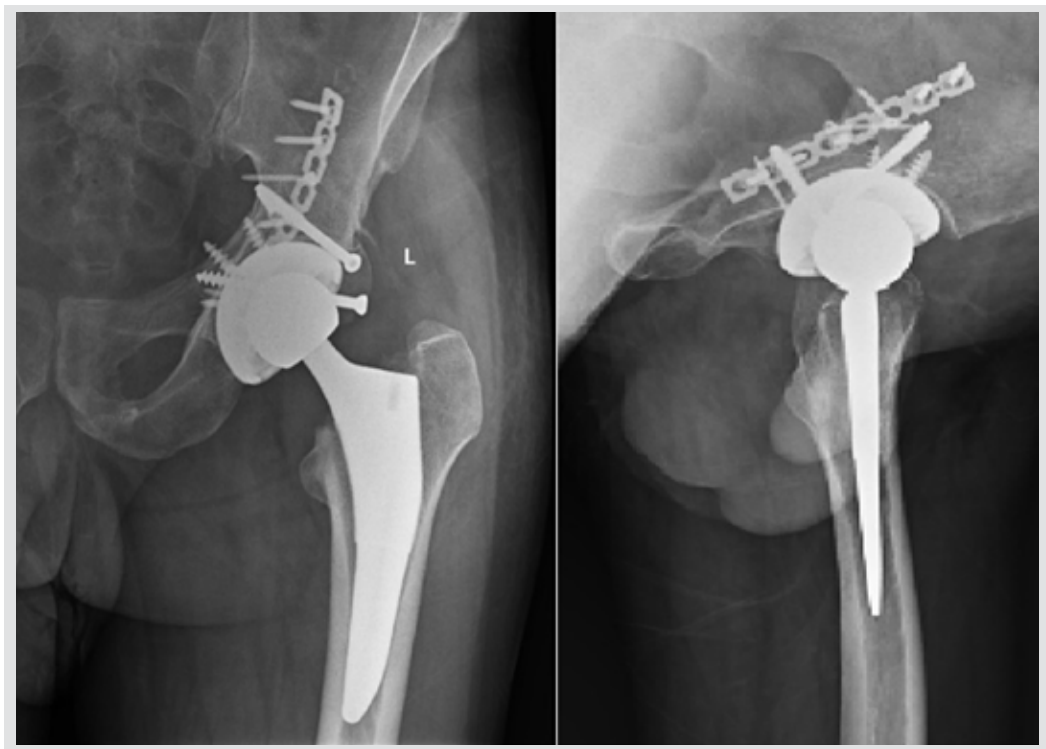

Figure 6: Final radiographs at 2-year follow-up showing stable well-fixed implant without any signs of loosening or 


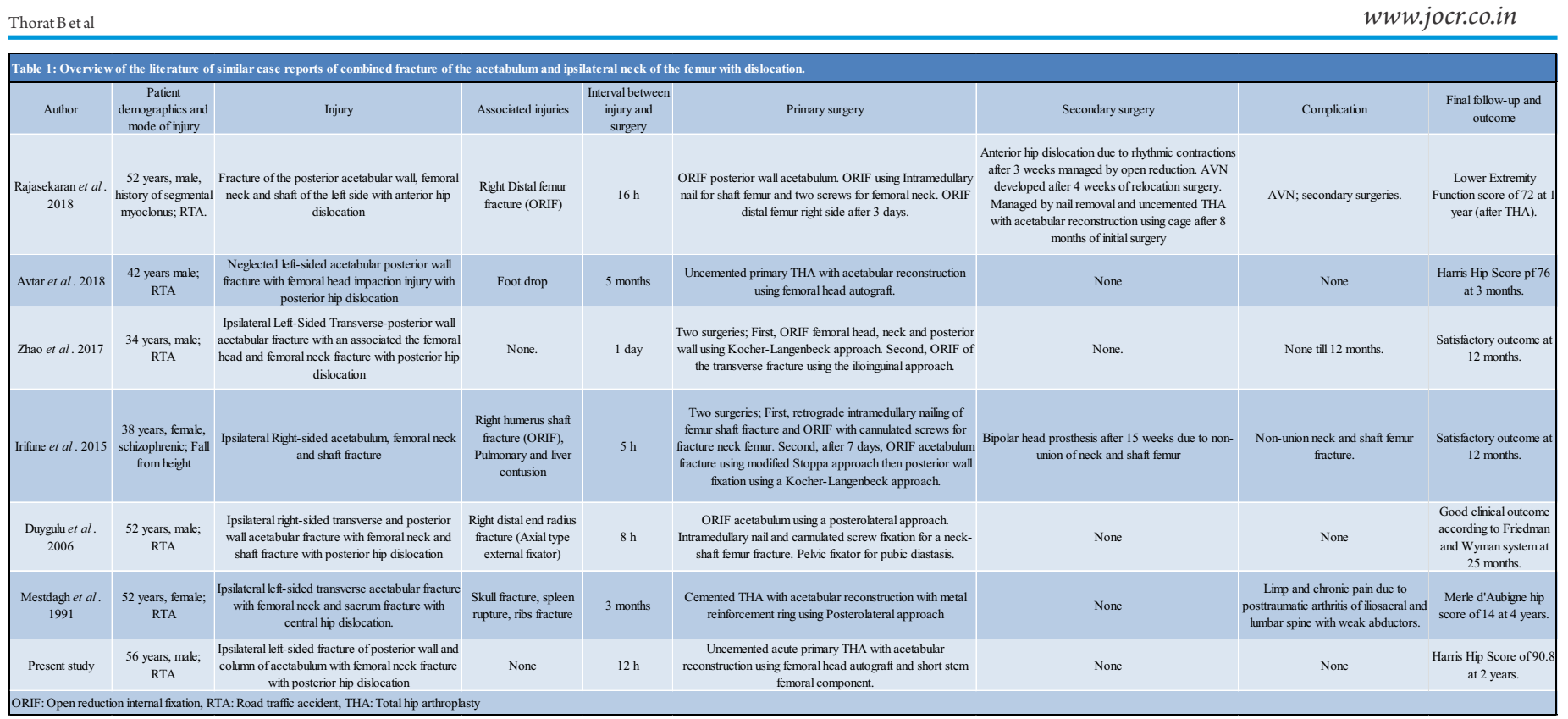

arthroplasty in acetabular fractures can be ascribed to technical difficulties, poor implant selection such as use of morselized allograft, bipolar prosthesis, cemented components with poor cementation techniques, or cup-cage construct to provide initial stability $[2,3,4,7,9]$.

Femoral head as structural auto or allograft has been widely used in case with deficient acetabulum due to dysplastic hip or previous acetabular fracture with non-union/mal-union, revision arthroplasty $[3,10]$. However, surprisingly little literature is available about acetabular reconstruction using femoral head structural autograft in acute primary THA for acetabular fractures in younger patients even though the conversion into arthroplasty after osteosynthesis remains high in such injuries [4]. Acetabular reconstruction using femoral head structural autograft and acute primary uncemented THA is a surgically demanding procedure in these cases and has been rarely reported in literature. It is an ideal definitive treatment option which facilitates post-operative recovery and early rehabilitation, thereby improving the patients' long-term functional outcome $[4,6,7]$.

Our case was unique and different owing due to complex and rare fracture pattern, use of porous short stem femoral component and definitive management by acute primary uncemented THA using femoral head as structural autograft for acetabular reconstruction, whereas, most of the previous studies report use of morselized/reamed femoral head graft or cages to fill the defect/cavity medial to the acetabular component $[3,4,6,7,9,10]$. The posterior approach was used in our case as this was the direction of dislocation and it gave easy access for sciatic nerve exploration and acetabular reconstruction, thereby, preserving the anterior structures from further iatrogenic injury to soft tissues. We encountered difficulty in reducing the medially displaced distal segment of hemipelvis, as the reducing force is to be applied against gravity. Hence, Steinman pin may be used to joystick the proximal fragment of the acetabulum while $\mathrm{K}$ wires for temporary stabilization before fixation $[3,6]$. Our case report, thus, emphasizes the importance of the development of standard classification system and management guidelines for such complexinjury patterns.

Outcomes of late THA after failed primary fixation is less favourable with increased complication rates in comparison to primary THA due to the extensive scarring and need for extensive soft- tissue dissection, the presence of occult infection in the bone and/or retained hardware and anatomic or structural abnormalities from bone defects or residual deformities $[6,7,8]$. Nowadays, use of highly porous metal components allows rapid biological ingrowth improving stability, and have has reported excellent clinical and functional outcomes in acute primary THA after acetabular fractures $[3,4$, 6].

A total of 6 six articles reporting on 4 four male and 2 two female patients aged between 34 and 52 years having posterior hip dislocation with ipsilateral fracture of femoral neck and acetabulum were reviewed (Table 1 ). The mechanism of injury included road traffic accidents $(\mathrm{n}=5)$ and falling from a height $(n=1)$. The complex nature of injury in our case is shown to be associated with a high chance of femoral head AVN. Two patients treated with primary THA had a satisfactory outcome without the need for revision surgeries. Out of four patients that underwent hip preservation surgeries, one patient developed anterior hip dislocation with AVN while another patient had femoral neck non-union, necessitating conversion into hip arthroplasty later on. Two patients needed metal reinforcement ring and acetabular cage for acetabular reconstruction during THA, while, acetabular reconstruction using the femoral head 
as autograft obviated the need for such implants in our case. We recommend the choice of an implant to be decided from a caseto-case basis.

Although satisfactory results could be expected after ORIF for patients with ipsilateral acetabular and femoral neck fractures without hip dislocation, we believe that uncemented primary THA with acetabulum reconstruction using femoral head autograft can be a good alternative for middle-aged to elderly patients having posterior hip dislocation with ipsilateral acetabulum and femoral neck fracture when there is a concern about non-union and AVN and avoids repeated surgeries $[6,7$, $8]$.

\section{References}

1. Tannast M, Mack PW, Klaeser B, Siebenrock KA. Hip dislocation and femoral neck fracture: Decision-making for head preservation. Injury 2009;40:1118-24.

2. Harris WH. Traumatic arthritis of the hip after dislocation and acetabular fractures: Treatment by mold arthroplasty. An endresult study using a new method of result evaluation.J Bone Joint Surg Am 1969;51:737-55.

3. De Bellis UG, Legnani C, Calori GM. Acute total hip replacement for acetabular fractures: A systematic review of the literature. Injury 2014;45:356-61.

4. Malhotra R, Gautam D. Acute total hip arthroplasty in acetabular fractures using modern porous metal cup. J Orthop Surg (Hong Kong) 2019;27:2309499019855438.

5. O’Toole RV, Hui E, Chandra A, Nascone JW. How often does open reduction and internal fixation of geriatric acetabular fractures lead to hip arthroplasty? J Orthop Trauma 2014;28:148-53.

6. Makridis KG, Obakponovwe O, Bobak P, Giannoudis PV. Total hip arthroplasty after acetabular fracture: Incidence of complications, reoperation rates and functional outcomes: evidence today. J Arthroplasty 2014;29:198390.

7. Mears DC, Velyvis JH. Acute total hip arthroplasty for selected displaced acetabular fractures: 2-12-year results. J Bone J Surg 2002;84A:1-9.

8. Kreder HJ, Rozen N, Borkhoff CM, Laflamme YG, McKee MD, Schemitsch EH, et al. Determinants of functional outcome after simple and complex acetabular fractures involving the posterior wall. J Bone Joint Surg Br 2006;88B:776-82.

\section{Conclusion}

Combination of posterior dislocation of the hip with Clinical Message

Although surgically challenging, an acute primary total hip arthroplasty with acetabular reconstruction using femoral head structural autograft can be a good alternative to ORIF in patients with complex acetabular fracture with ipsilateral neck femur fracture to avoid secondary procedures in near future.

9. Hamlin K, Lazaraviciute G, Koullouros M, Chouari T, Stevenson IM, Hamilton SW. Should total hip arthroplasty be performed acutely in the treatment of acetabular fractures in elderly or used as a salvage procedure only? Indian J Orthop 2017;51:421-33.

10. Harris $\mathrm{WH}$, Crothers $\mathrm{O}$, Oh I. Total hip replacement and femoral-head bone-grafting for severe acetabular deficiency in adults.J Bone Joint Surg Am 1977;59:752-59.

11. Rajasekaran RB, Jayaramaraju D, Palanisami DR, Perumal $\mathrm{R}$, Shanmuganathan R. Ipsilateral acetabular fracture with displaced femoral head and femoral shaft fracture: A complex floating hip injury. Case Rep Orthop 2018;2018:4937472.

12. Avtar R, Khanna V, Kumar K. Neglected posterior acetabular wall fracture managed with uncemented total hip arthroplasty with posterior wall reconstruction by femoral head autograft: A case report. Int J Res Orthop 2018;4:527.

13. Zhao B, Li H, Yan J, Han LR, Yang XF. Pipkin Type III femoral head fracture-dislocation combined with complicated acetabular fracture. Medicine 2017;96:e9214.

14. Irifune $\mathrm{H}$, Hirayama S, Takahashi N, Narimatsu E. Ipsilateral acetabular and femoral neck and shaft fractures. Case Rep Orthop 2015;2015:351465.

15. Duygulu F, Calis M, Argun M. Unusual combination of femoral head dislocation associated acetabular fracture with ipsilateral neck and shaft fractures: A case report. J Trauma 2006;61:1545-8.

16. Mestdagh H, Butruille Y, Vigier P. Central fracturedislocation of the hip with ipsilateral femoral neck fracture. J Trauma 1991;31:1445-87. 


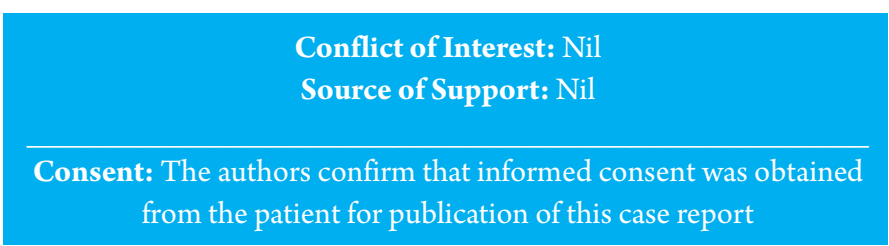

How to Cite this Article

Thorat B, Singh A, Arshad M, Salokhe S, Mavani R. "Acute Primary Total Hip Arthroplasty for Combined Posterior Acetabulum Fracture with Ipsilateral Associated Posteriorly Dislocated Femoral Head with Femoral Neck Fracture. Using of Femoral Head as an Autograft Would be an Advantage". Journal of Orthopaedic Case Reports 2021 April;11(4): 85-90. 